\title{
VBMF Regimen
}

National Cancer Institute

\section{Source}

National Cancer Institute. VBMFRegimen. NCI Thesaurus. Code C10176.

A chemotherapy regimen consisting of vincristine, bleomycin, methotrexate, and fluorouracil that may be used in the treatment of head and neck cancer. 